\title{
Screziature del Bello
}

\author{
di Gabriele Scaramuzza
}

gabriele.scaramuzza@gmail.com

\begin{abstract}
Rather than discussing beauty or ugliness as metaphysical categories I do prefer here to consider individual artistic worlds, and to defend the values in which I recognise myself as reader and enjoyer, in the various ways in which this can be articulated. Worlds in which aesthetic positivity and negativity are in tension, each resolving this dialectic in its own way. That's why my paper doesn't analyse Beauty or Ugliness tout-court. I do rather consider their dialectical intertwining and reflecting in specific works of art: The Idiot by Dostoevskij, the librettos to Wagner's Lohengrin and Verdi's Rigoletto, as well as Fatelessness by Imre Kertész. I intend to show how a negative experience can be transfigured in the structure and language of these writers and musicians.
\end{abstract}

Keywords: Ugliness, Beauty, Fate, Redemption

Ai discorsi sulla Bellezza tout-court e sulla sua problematicità ${ }^{1}$ preferisco qui la considerazione di singoli mondi artistici, e la difesa in essa dei valori in cui mi riconosco, nei vari modi in cui questo può articolarsi. Mondi che vedono in tensione la positività e la negatività estetica, e risolvono ognuno a modo proprio questa dialettica. Neppure Hegel abolisce il dualismo, lo mette in tensione e, è vero, ne postula uno scioglimento.

\section{La "bellezza" dell'Idiota}

Prendiamo innanzitutto il caso di un romanzo che resta tra i miei preferiti: L'Idiota. In che senso può esser qualificato come "bello"? Non a caso la riuscita

\footnotetext{
${ }^{1}$ Questo saggio è in buona parte tratto dal mio Scelte, Mimesis, Milano 2021, pp. 47-82. Anche qui preferirei tener distinti (seguendo in questo il pensiero di Dino Formaggio) gli ambiti estetico e artistico; e parlare, anziché genericamente di bellezza, di un termine che designa diversi valori: innanzitutto la compiutezza artistica e la densità estetica, con tutte le variazioni che si possono loro annettere, e la nostra tradizione ha indicato come "categorie estetiche" e/o "modificazioni del bello".
} 
artistica di Dostoevskij è difesa da Bachtin, ma la sua scrittura è vivacemente contestata da Nabokov. Dostoevskij scrive a un'amica che il principe Myshkin è uomo "positivamente buono», ma Vittorio Strada traduce «totalmente bello»; il russo dunque si presta a entrambe le cose. Non è facile, e spesso riesce melenso scrivere di eroi buoni, Dostoevskij lo sa bene: la storia dell'Idiota non è la storia della bontà, né un'apologia della bontà, tanto meno della «bellezza che salva il mondo», come Myshkin pur afferma. È piuttosto la storia della disfatta della bontà-bellezza, vista nelle laceranti aporie di cui è vittima ${ }^{2}$. Accanto a Cristo, l'altra figura ispiratrice del romanzo è Don Chisciotte essere "buono" anche lui; ma la sua bontà è costantemente messa in scacco dal ridicolo e dal fallimento che lo perseguita.

Rocco e $i$ suoi fratelli di Luchino Visconti eredita non poco da questo mondo, cui va aggiunta La Traviata. Rocco rinvia a Myshkin: anche la sua bontà provoca disastri. Le diverse, enigmatiche bellezze di Nastas’ja, della Nadia viscontiana, di Violetta, non salvano nulla e nessuno ${ }^{3}$ : loro comuni sono l'atmosfera di prostituzione, e l'ansia di riscatto, ben colte da Myshkin e da Rocco; comune è la caduta finale: l'assassinio compiuto da Rogozin e da Simone. Fortissime analogie sono evidenti tra la folle scena finale, a delitto compiuto, tra Rogozin e Myshkin e quella tra Simone a Rocco.

L'Idiota risulta da una polifonia (che è contrasto, non ipocrita composizione "buonista") di mondi, valori, e anche stili diversi, che danno carne ai differenti contesti in cui conflittualmente vive la bellezza-bontà. Ma anche è la enigmatica riproposta di valori alti, anche se irrealizzabili; dire la sua "bellezza" significa mettere in gioco una molteplicità di termini e di temi, che nel solo bello non avrebbero voce.

\footnotetext{
2 «O don fatale, o don crudele / che in suo furore mi fece il cielo! / tu che ci fai sì vane, altere, I ti maledico, o mia beltà», canta la Principessa d'Eboli nel terzo atto del Don Carlo verdiano. ${ }^{3} \mathrm{Nel}$ contesto delle nostre conversazioni a Corrente rinvio alla relazione di Roberto Diodato, che ha per titolo "La bellezza, qualsiasi cosa sia, non salverà il mondo" [on-line], su Fondazione Corrente Onlus - Home / Facebook. Pubblicato il 10 novembre 2021, consultato il 10 dicembre 2021. Disponibile all'indirizzo: https://www.facebook.com/1408683729436034 /videos/319028896324051/?_so_=channel_tab\&_rv_=all_videos_card.
} 
Tra i mondi che mettono in tensione la positività e la negatività estetica e/o artistica vi è quello del teatro musicale; un universo estremamente complesso: molte realtà vi si intrecciano: da sonore a visive a verbali. Mi soffermo su due opere lontane tra loro: Lohengrin e Rigoletto. A cosa ci si deve appigliare per delinearne un valore, ciò sulla cui base diciamo che un'opera "è bella", "mi piace", "la preferisco a"...? Alcuni tratti di queste celebri opere mi offrono materiali di riflessione: appartengono entrambe al mondo della bellezza, o della sua assenza. Procedo per considerazioni sparse, relative solo ad alcuni momenti di esse.

\section{Lohengrin}

Se si vuol parlare di bellezza, o del suo contrario, come si può motivare l'uso di simili termini a proposito di un dramma musicale, per sua natura così stratificato? Il discorso non può arrestarsi a una semplice affermazione (o, meglio, esclamazione): di fatto si rifrange in una miriade di considerazioni, non tutte sullo stesso piano. Prendiamo dunque innanzitutto qualche tratto del Lohengrin o, più esattamente, del testo del Lohengrin.

Il terzo atto, verso la fine, vede il crollo di ogni certezza, della fedeltà tra Lohengrin e Elsa, del loro amore, fondato sulla fiducia nel lato sensibile ("estetico": in senso proprio dunque) della vita: sul darsi sensibile delle cose e sui presentimenti in esso vivi. Di qui la richiesta di Lohengrin: «mai devi domandare». Condizione posta fin dall'inizio da Lohengrin, e vero e proprio tema che muove tutta la vicenda, arma che nelle mani di persone ostili alla fine la distruggerà. Come conferma Quirino Principe, «Il vero nucleo drammatico dell'opera è il divieto di porre la domanda, e tutto il resto [...] ne deriva per concatenazione» ${ }^{4}$.

\footnotetext{
${ }^{4}$ Q. Principe, "Il bianco e l'argento di Lohengrin", in Lohengrin, Programma di Sala, Edizioni del Teatro alla Scala, Milano 2006, p. 157. Da leggere è anche, su un diverso versante, il brano dedicato da Charles Baudelaire al tema della domanda vietata in C. Baudelaire, Richard Wagner, tr.. it. di L. Merlini, con prefazione di G. Macchia, Passigli, Firenze 1983, pp. 67-70.
} 
Elsa rompe questo patto perché vuole certificazioni fattuali «intellettive, ragionevoli», non si accontenta di quelle sensibili: vuol sapere il nome, donde venga, dove vada, cosa voglia Lohengrin... - e perde l'amore. Istigata da Ortrud $^{5}$, vero e proprio Jago al femminile, Elsa chiede, non si accontenta di quanto la «pura presenza» di Lohengrin le offre. Non è così anche per Otello? soccombe perché ha perso ogni fiducia nella presenza sensibile/sensuale di Desdemona, nella verità dei suoi occhi, delle sue carezze... D’altronde il loro amore era nato dalla pietà più che da una reciproca attrazione sensibile/sensuale, come si evince dal duetto finale del primo atto verdiano: «E tu m'amavi per le mie sventure / Ed io t'amavo per la tua pietà. / Ed io t'amavo per le tue sventure / E tu m’amavi per la mia pietà».

Al primo accennarsi della richiesta del nome da parte di Elsa Lohengrin risponde con parole altrettanto "belle", nella mia ottica, quanto quelle di Winterstürme (le tempeste invernali che cedono il passo all'incanto della luna primaverile, sulla bocca di Siegmund e Sieglinde nel primo atto di Die Walküre):

Non respiri anche tu con me i dolci profumi?
Con quale incanto inebriano i sensi!
Misteriosamente essi ci giungono attraverso l'aria,
senza domandare io mi concedo al loro incanto
[...] Non appena il mio occhio ti scorse, il mio cuore ti comprese;
mai svanirà il tuo fascino
se resti lontana da ogni dubbio ${ }^{6}$.

A crollare è la fiducia nel mondo sensibile, nella verità della Sinnlichkeitche accomuna sensibilità, sensualità, ed emotività. Questo mondo ha una

\footnotetext{
${ }^{5}$ Se le false accuse, l'inganno e l'astuzia di Telramund non riescono, esclama Ortrud, restano la violenza verso Lohengrin e Elsa: «Missglückt's / so bleibt ein Mittel der Gewalt»; restano «der Rache süsse Wonnen», le dolci voluttà della vendetta. Che mondo si configura in questo? Civilmente, eticamente, politicamente.

${ }^{6}$ Queste e le seguenti citazioni sono tratte dalla traduzione di Q. Principe di Lohengrin, cui fa seguito Wagner e noi dello stesso Principe, Jaca Musica, Milano 2012. Per il brano ora citato v. anche F. Liszt, Lohengrin e Tannhäuser di Richard Wagner (1854), tr. it. di R. Morello, introduzione di P. Isotta, Mondadori, Milano 1983, p. 43. In generale cfr. le pagine dedicate a Lohengrin da C. Dahlhaus, I drammi musicali di Richard Wagner, tr. it. a cura di L. Bianconi, Marsilio, Venezia 1984, pp. 49-63.
} 
certezza vissuta che lo pone al di sopra delle certezze "discorsive": le raisons $d u$ coeur, l'intelligenza primaria...

Nella figura di Lohengrin fa pensare la discendenza da Parsifal. Invece di vedere Lohengrin come "puro folle", devoto al Graal, e portatore di quei valori nel Brabante, non lo si potrebbe vedere come chi tenta la fuga da un mondo mistico-sensuale asfittico, cerca la salvezza ad Anversa, riscatta la dimensione sensibile, terrena, e viene ricondotto nella prigione da cui proviene dall'insensatezza di Ortrud, e di Elsa istigata da essa? Se veramente desiderasse una sorta di incendio del mondo del Graal, simile a quello del Walhalla nel finale del Ring? 亡̀ una supposizione, non so quanto fondata, certo.

Per contro, una domanda resta sospesa: il privilegiamento della verità "estetica" è ben comprensibile nel caso dell'innamoramento, dei rapporti affettivi. Elsa e Lohengrin hanno avuto il presentimento l'uno dell'altra, si sono attesi, senza neppure essersi conosciuti (Elsa lo ha solo sognato). Elsa lo ha chiamato in soccorso senza nulla sapere, e Lohengrin è corso in suo aiuto sfidando Telramund, senza nulla chiedere, senza voler conoscere le sue ragioni, senza indagare. Ma spostando il punto di vista: Lohengrin è atteso non solo da Elsa che lo ama, ma anche dal Brabante tutto, come conduttore che lo porterà alla vittoria contro il loro nemico. Come può fondarsi su un'intuizione inesprimibile, su un indiscutibile ma vago presentimento, il fatto che il Brabante (tedesco beninteso) confidi in Lohengrin come suo Führer? il termine ricorre almeno due volte, nel senso di condottiero, duce, non in senso nazista ovviamente: Wagner muore cinquant'anni prima della Machtübernahme. Non è proprio possibile sapere come si sarebbe comportato nei confronti della Shoah; avrebbe condiviso i deliri di Winifred, dopo quelli di Cosima (per sua fortuna defunta nel 1930)?

E ancora. Si fonda sulla segretezza e sull'inesprimibile mistero il diritto al potere? Le conseguenze politiche sarebbero devastanti. Contrastano ogni democrazia, prefigurano uno stato totalitario, in cui contano obbedienza e 
fedeltà, sottomissione. Inoltre, non aveva ogni diritto Elsa di chiedergli? perché l'amore pone per condizione il non sapere, è nemico del dialogo, deluso della ragione? Non è un inganno, e nefando, anche questo?

Continuiamo a leggere: «Dov'era colui che è stato inviato da Dio / per la gloria e la grandezza del Brabante?». Il Re e i suoi seguaci del Brabante lo attendono "ansiosi di combattere / da te guidati, certi della vittoria»; e il nemico sta a Est! Lohengrin si ritrae però: «Non come alleato sono venuto, / ma come accusatore voglio essere ascoltato».

Poi chiarisce raccontando la propria storia: "In un paese lontano...». Si giustifica, accusa (colpa è avergli chiesto il suo nome, aver preteso che svelasse il suo mistero). Ma promette: "Anche nei giorni più lontani le orde orientali / non entreranno mai più vittoriose in terra tedesca». Torna al Graal, ma l'effetto della sua venuta è l'arrivo del vero Führer, Gottfried il Duca di Brabante (fratello di Elsa) che lo porterà alla vittoria: «Seht da den Herzog von Brabant, / Zum Führer sei er auch ernnant». Come stupirsi se Hitler ha sostenuto che «chiunque voglia comprendere la Germania nazionalsocialista deve conoscere Wagner»?

Da considerare è anche il linguaggio letterario di Wagner, quel suo tedesco in cui ricorrono termini nazionalistico-militaristi: spada, fedeltà, contesa (Giudizio di Dio addirittura), sudditanza, tedesco... Termini che sembrano, secondo taluni, rinviare alla LTI studiata da Viktor Klemperer, più che al tedesco che amiamo in Goethe, in Hölderlin, in Kafka, in Wittgenstein o in Rilke... Certo bisognerebbe avere quella sensibilità che solo chi è madrelingua possiede per poter valutare il linguaggio di Wagner. Un'amica bavarese, esperta e nota traduttrice dall'italiano in tedesco, mi parla dei suoi testi (riferendosi in particolare al Ring) come scritti «in uno stile falso e bombastico». I testi di Wagner, a quanto so, non sono universalmente 
apprezzati. Personalmente ammiro Winterstürme; apprezzo lo stile onomatopeico, quello stridio tra le parole, dell'inizio dell'Oro del Reno.... Nel Lohengrin, come altrove in Wagner, noto la frequenza nelle didascalie di termini espressionisticamente tesi, inquietanti anche nella gioia: "solenne orrore", "con sempre maggior violenza", "esaltata animazione”, passione, estaticità, eccitata curiosità, paralisi da spavento, o da stupore, teso silenzio, solenne eccitazione, esaltazione parossistica... In ogni caso è da sottolineare che le parole vanno viste alla luce di quello che la musica se ne fa. Le parole attendono la musica, sono scritte in sua funzione; e nella funzione che entrambe assumono nel dramma intero, che è Wort-Ton-Drama, appunto.

Decisiva resta dunque la musica; solo essa può verificare ogni ipotesi messa in campo. Ma non cancella il resto: ci reagisce se mai. Soggettivamente, tra i momenti musicali esteticamente più alti annovererei, oltre al preludio, i noti Einsam in trüben Tagen, In fernem Land, altri momenti, tra cui da ultimo mi ha preso il motivo (il più toccante nella mia ottica) che, nella seconda scena del secondo atto, accompagna Elsa che canta: Durch euch kam er gezogen...

Cosa dice la musica del Lohengrin, come tratta questa vicenda intrisa di nazionalismo e di violenza, che si affida al carisma di un condottiero che si sottrae a ogni domanda, la punisce anzi? Non è Wagner stesso ad affidare alla musica il senso del finale dell'intero Ring, senza cercar parole per rispondere a chi glielo chiede? Solo l'ascolto può far conoscere cosa Wagner musicalmente se ne fa, della sua visione dichiarata del mondo.

Il "Non-bello" di Lohengrin sta a mio avviso in quanto configura un'atmosfera nazionalista e bellicista. Si sa quanto Hitler amasse Wagner, e dapprima proprio Lohengrin: dopo aver assistito a Linz per la prima volta al Lohengrin, scrive: «Di colpo mi trovai come rapito. La mia esaltazione giovanile per il maestro di Bayreuth non conobbe limiti», e come noto non si 
esaurì mai. Non poco di $\mathrm{Il}$ giudaismo nella musica ${ }^{7}$ torna (pur senza esser nominato) in Mein Kampf. Ci sono stati mutamenti di prospettive, talvolta rivolgimenti, nella vita di Wagner; non è mutato tuttavia il suo antisemitismo - che resta pur sempre una parte della sua personalità, non meno di quanto lo sia la sua arte. Ci sono tracce anche nel Lohengrin dell'antisemitismo di Wagner? A parere di Quirino Principe «Lohengrin non rientra nelle discussioni sull'antisemitismo di Wagner, mentre vi rientrano Der fliegende Holländer, Der Ring des Nibelungen, Die Meistersinger, Parsifal» ${ }^{8}$. Che siano ebrei Ortrud e Telramund non è detto. Certo però sono ipocriti, perfidi, astuti, infidi, ingannatori... E l'atmosfera in cui operano è totalitaria, illiberale, e questo può indurre tentazioni antisemite.

L'ascolto di Wagner mi aveva portato a rimuovere il libello del 1850, pervicacemente riedito, non modificato nella sostanza e allargato, anzi confermato purtroppo, nel 1869 - a Tristan, Meistersinger e buona parte del Ring conclusi. Si sarebbe tentati di metterlo su di un piano analogo a Bagatelle per un massacro (scritto da chi pur ci ha lasciato uno dei romanzi più belli del '900: Viaggio al termine della notte), ai Protocolli dei Savi di Sion, al Mein Kampf... Qui sono anticipati la Bayreuth nazista, l'antisemitismo virulento di Cosima e soprattutto di Winifred, Hitler sul campo... Winifred, la nuora di Wagner, caldeggiò la Machtübernahme; fu grande amica di Hitler, fu ben viva durante la Shoah, morì nel 1980 - si sa.

\footnotetext{
7 Pubblicato nel 1950, lo stesso anno in cui fu dato per la prima volta a Weimar Lohengrin. Del 1951 è Rigoletto (preceduto da Luisa Miller e Stiffelio). Si tratta dunque di eventi pressocché coevi.

8 Q. Principe, "Il bianco e l'argento", cit., p. 157, sembra ritenere «un discorso di secondo piano, che ha dato esiti sovente volgari», quello che coinvolge l'atteggiamento di Wagner verso gli ebrei - se bene intendo.
} 
Un misto di fascino e di ripulsa mi ha messo a disagio con me stesso di fronte a Wagner; o quanto meno col me stesso che non sa del tutto scindere le opere d'arte dalla persona che le ha create. Sappiamo: c'è un'emergenza dell'opera d'arte, e dell'autore di essa, rispetto alla restante "vita". Ma davvero l'autore può essere avulso dalla personalità "in carne ed ossa"? In certa misura lo è; ma non del tutto9. L'emergenza dell'opera è indispensabile per mettere in rilievo aspetti dell'opera che altrimenti resterebbero in ombra. Ma non si può contrabbandare una differenziazione metodica per una differenza ontologica o magari assiologica.

Il mio (e non solo mio) modo di fruire Wagner mi porta a chiedermi come mai in lui possano convivere dati così inconciliabili come il fascino dei drammi musicali e l'antisemitismo. Sono entrambi dimensioni importanti della sua esistenza, possono avere variabili rapporti di interdipendenza. Ma non possono venire semplicemente poste in insanabile conflitto, o in rapporto di reciproca esclusione; altrimenti si dovrebbe parlare di schizofrenia in Wagner, e in noi che lo ascoltiamo con partecipazione. Forse si può ipotizzare nello stesso Wagner un fondo antecedente ogni separazione; e che in esso possiamo trovare una nostra personale "pacificazione" nell'ascolto.

\section{La donna è mobile}

Wagner e Verdi sono contemporanei, ma sono molto diversi, e non solo per le loro musiche. Diversi gli ambienti, diversi i modi di vivere il loro tempo, di reagirvi con le loro opere; diversi i loro caratteri, le visioni del mondo. Quanto consoni dopotutto Verdi e la sua musica, quanto stridenti la persona Wagner e i suoi drammi! E questo si avverte bene già nell'ascolto. Tra quanto più mi urta in Wagner, accanto al suo narcisismo, alla sua presunzione, è il disprezzo

\footnotetext{
${ }_{9}^{9} \mathrm{Mi}$ sono annotato pro domo mea una frase di Sandro Mancini: «ritengo che la verità sia indivisibile, e altrettanto indivisibile sia la persona».
}

Materiali di Estetica - N. 8.2: 2021, Pagina 370 
verso altri, in particolare verso Verdi. Disprezzo diffuso in molti (wagneriani e no), ma non condiviso da wagneriani di pur alto livello.

Non so cosa abbia motivato i Wagner ad andare a sentire il Requiem verdiano a Vienna (forse Bülow già sulla via del pentimento?); ne ricavarono comunque un'impressione malevola, come riferisce Cosima nella sua “Tagebuchnotiz” del 1875: «Ein Werk, worüber nicht zu sprechen entschieden das Beste ist». Il nome di Verdi non compare ovviamente in La mia vita, non manca però una stoccata contro Il Trovatore ${ }^{10}$. Nell'edizione tedesca in mio possesso del Mein Leben, accanto al Trovatore, troviamo versi che riguardano anche il Don Carlos, messo tra le opere «denen gerbten wir erst das Fell» [conciato per le feste], immagino nell'edizione parigina del 186711. Cosima Wagner certo condivideva le idee del marito circa Verdi. In una lettera a Ludwig Schemann dell'aprile del 1996, da Bayreuth, indirettamente accenna (e non certo in modo lusinghiero) a Rigoletto, parlando della nota parafrasi di Liszt: «Di una delle fantasie verdiane, uno scrittore francese ha detto: "C'est un grandseigneur [Liszt] présentant un mannequin [cioè Rigoletto]"»12.

Vorrei aggiungere per contro quanto Verdi disse in un incontro con Felix Philippi a Milano nell’inverno 1898-1899:

L'opera che ha sempre suscitato la mia più grande ammirazione è il Tristano. Di fronte a questa titanica costruzione resto sempre con immenso stupore: non si riesce a credere che l'abbia concepita e scritta un essere umano. Penso che il secondo atto sia una delle creazioni più sublimi dello spirito umano nel campo dell'invenzione musicale, in particolare per la tenerezza e la sensualità dell'espressione musicale e per la geniale strumentazione. Questo second'atto è meraviglioso... semplicemente meraviglioso ${ }^{13}$.

\footnotetext{
${ }^{10} \mathrm{R}$. Wagner, La mia vita, con un'equilibrata introduzione e con la traduzione di M. Mila, EDT, Torino 1983, pp. 466. Non ricordo dove ho letto, e sarebbe da verificare, che Wagner tenne un rispettoso silenzio verso La Traviata.

${ }^{11}$ R. Wagner, Mein Leben, Schünemann, Bremen 1986, vol. II, p. 351; p. 194 per Il Trovatore. 12 C. Wagner, La mia vita a Bayreuth 1883/1930, a cura di D. Mack, tr. it. di U. Gandini, Rusconi, Milano 1982, p. 424.

${ }_{13}$ M. Conati, Verdi. Interviste e Incontri, EDT, Torino 2000 (ediz. riveduta), pp. 367-368; e vd. le pp. 363-369. La rievocazione di questo incontro fu pubblicata dal «Berliner Tageblatt» il 13 luglio del 1913.
} 
Certo Verdi parlava a un tedesco, e wagneriano, ma il tono delle sue parole non può essere solo un omaggio all'ospite; e mostra una conoscenza non superficiale del Tristan.

Rigoletto dunque: atto terzo, scena nona; per la terza volta il Duca canta La donna è mobile. Molti ostacoli si oppongono a definirla "bella": la banalità dei versi e della melodia, la situazione, in cui interagiscono la deformità del protagonista, il sacco, il paesaggio angosciante, dominato da un fiume cupo, gli echi del temporale e del delitto antecedenti. Eppure la scena, che sfocia in un finale che è anche la toccante aria di Gilda morente, è "bella", nel senso di artisticamente riuscita, indubbiamente.

La donna è mobile è per solito disprezzata per la sua modestia sul piano estetico; in un'ottica propriamente artistica per contro viene riscattata la sua "verità", la sua efficacia, drammaturgica. Ha una sua funzione non trascurabile nell'insieme del terzo atto di Rigoletto: dalla prima volta in cui viene cantata in tono scanzonato e derisorio, alla seconda in cui il Duca la spegne nel breve sonno, fino al suo ricomparire, raggelante, verso il finale. Dove Rigoletto crede che si sia compiuta la sua vendetta, e sente invece la maledizione incombere su di sé: il canto attesta che il Duca è vivo, nel sacco si trova la figlia morente.

Analogamente, nel finale del secondo atto esplode il Sì, vendetta: un "non bello", enfatico nella mia ottica, sul piano estetico; riscattato tuttavia dalla funzione artistica che assume.

Mi ha opportunamente scritto Tiziana Canfori:

Io ho sempre amato questo punto dell'opera, soprattutto "Tutte le feste al tempio" e la parte centrale del duetto. "Vendetta" è enfatico, ma entusiasmante, quindi perdonatissimo. Ciò che nasce dal personaggio di Rigoletto mi pare sempre appassionante. Certo, la mite Gilda nulla può con le sue proposte di perdono contro la furia del padre, ma mi piace l'idea che la sua fretta nasca da un desiderio urgente anche per lei: quello della comprensione e del perdono anche per se stessa. Ovviamente la tonalità è la stessa, la bem. magg., ma ovviamente 
i due non possono cantare le stesse note, trovandosi in tessiture diverse. Succede che Gilda canta la stessa frase di Rigoletto in re bem. magg,, alla sottodominante. Prassi normale, in un duetto. Non andrei a cercare simmetrie fra i due personaggi: certo Gilda non è Rigoletto e non ha la sua rabbia, ma qui la verità travolge entrambi e li trascina. Gilda non fa la femminuccia, voglio dire, ha da esprimere un turbamento di fuoco, anche lei. Dopo il racconto di Gilda c'è il momento più intimo del rapporto padre figlia, con quel "piangi" che dice tutto il rudimentale affetto di Rigoletto, riconosciuto da Gilda come l'espressione di un "angel consolator". In questo punto due affetti feriti si toccano e si riconoscono. Bellissimo. Da qui, dopo il passaggio di Monterone, sgorga l'ira battagliera e vendicatrice di Rigoletto, che tutto travolge, anche i timori della figlia. Lo scarico d'energia alla fine è verdianissimo e sublime, soprattutto con un mi bemolle perfetto del soprano.

\section{"Bellezza" del racconto della Shoah?}

Imre Kertész, in Essere senza destino ${ }^{14}$, narra con tono inusitato, sorprendente, originale davvero, le proprie atroci traversie nei lager nazisti, e le incertezze della liberazione: i difficili reincontri, le prime nontestimonianze, le difficoltà, non lievi, del reinserimento a Budapest, col "socialismo reale" all'orizzonte. Con occhio svagato, da Alice nel paese degli orrori, in Essere senza destino l'autore prende tutto come "ovvio", "naturale" (sono termini che ricorrono spesso nel testo); opera un evidente understatement dell'orrore attraversato; ne scorge persino tratti positivi, persino umoristici. A proposito della tecnica di straniamento usata, in Dossier $K$. leggiamo: «L'estraneità del linguaggio di Essere senza destino si può spiegare unicamente con l'estraneità della materia e del narratore» ${ }^{15}$.

Effetti amaramente ironici, stravaganti, d'un umorismo cupo, sono presenti qua e là. Esemplificando, macabro-grottesca, surreale, è la descrizione dello spettacolo "ameno" allestito davanti alle camere a gas: «avevo l'impressione che fosse un specie di burla, uno scherzo goliardico o

${ }^{14}$ I. Kertész, Essere senza destino (1975), tr. it. di B. Griffini, Feltrinelli, Milano 2004.

15 I. Kertész, Dossier K. (2006), trad. di M. D’Alessandro, Feltrinelli, Milano 2009 pp. 157-158. 
qualcosa del genere»16. Dello sconcertante modo di essere di Kertész testimoniano anche le pagine finali di Essere senza destino:

persino là, accanto ai camini, nell'intervallo tra i tormenti c'era qualcosa che assomigliava alla felicità. Tutti mi chiedono sempre dei mali, degli "orrori": sebbene per me, forse, proprio questa sia l'esperienza più memorabile. Sì, è di questo, della felicità dei campi di concentramento che dovrei parlare loro, la prossima volta che me lo chiederanno ${ }^{17}$.

Poco sopra tuttavia parla della propria impossibilità di dimenticare gli orrori (cosa che qualcuno gli consiglia); e per converso dichiara, tra i presenti «piuttosto sbalorditi»: «io non mi sono accorto degli orrori». Uscendo, dopo l'incontro-scontro coi conoscenti appena rientrato a Budapest, nell'animazione delle vie al tramonto - la sua "ora preferita» anche al lager, dove «in un certo senso la vita era più pura, più frugale» - Kertész evoca atmosfere poetiche, colori struggenti, ricorre perfino a termini quali "infinito", "nostalgia", "sguardi più miti"; e scrive: «per la prima volta, adesso pensai [ai miei compagni di prigionia] con un piccolo rimprovero, con una specie di affettuoso rancore». E nonostante tutto restano vivi nei detenuti, e in Kertész sopravvissuto, la domanda circa il senso della loro esperienza; un desiderio di riconoscimento, di gentilezza, e la voglia di vivere. Soprattutto resta l'impossibilità non solo ad abituarsi anche nel ricordo al tipo di vita attraversato, la difficoltà estrema a capire.

Essere senza destino è dunque interamente dedicato alla diretta esperienza della Shoah del suo autore; nel successivo Diario dalla galera (e anni di galera sono quelli dei lager, ma anche quelli del cosiddetto "socialismo reale") troviamo la cronaca del periodo in cui, in Ungheria, Essere senza destino fu scritto, tra il 1961 e il 1973. Insieme troviamo una spiegazione illuminante

\footnotetext{
${ }^{16}$ Ivi, p. 96.

17 I. Kertész, Essere senza destino, cit., pp. 214-220. Di «eterna attualità» (accompagnata tuttavia da un «così pare») di queste pagine Kertész parla in L’ultimo rifugio. Romanzo di un diario (2014), trad. di M. Sciglitano, Bompiani, Milano 2016, p. 13; e a p. 221: «Ho letto le ultime pagine di Essere senza destino, e anche io sono rimasto sorpreso dalla chiarezza del testo, dalla sua classicità, dalla sua forza».
} 
del titolo enigmatico: «Cosa intendo per destino? In ogni caso la possibilità della tragedia». La vita nel totalitarismo decreta la fine della tragedia: se «noi viviamo come realtà la determinatezza che ci viene imposta, invece che la necessità derivante dalla nostra libertà relativa, questo io lo chiamo assenza di destino». Cade ciò che al destino è coessenziale, e cioè "che l'elemento di determinazione sia sempre in opposizione alle nostre idee e predisposizioni naturali»; manca il tragico. Ciò che «ha reso possibile Auschwitz», per converso ha reso impossibile «realizzare l'unica risposta adeguata a questo crimine unico al mondo: la catarsi». E ancora:

Hitler cercava di far apparire come "un inevitabile destino" ciò che inevitabile non era per niente e che anzi [...] non sarebbe dovuto proprio succedere. Questa è l'esperienza dell'essere senza destino, su un altro piano. Nel totalitarismo tutto accade sotto il segno del destino e della fatalità. Queste espressioni sono tenute a coprire il nulla, il completo Nulla che tuttavia produce montagne di cadaveri, devastazione e infamia.

È nota la frase pronunciata dal nostro "uomo della provvidenza" nel discorso dal balcone di Palazzo Venezia il 10 giugno 1940: «Un’ora, segnata dal destino, batte nel cielo della nostra patria». In altri termini, il totalitarismo induce a introiettare quasi fosse un dato di natura, inscalfibile, una realtà storica frutto di una decisione scellerata, e imposta. Sintomatico è che «evento senza destino» ${ }^{18}$ per Kertész è anche il crollo sovietico e la conseguente cosiddetta "liberazione", con l'orbanismo alle porte.

In Fiasco ${ }^{19}$, pubblicato nel 1988, erano state descritte "le circostanze della nascita" di Essere senza destino; e sono riportate le ragioni, sintomatiche, con cui questo romanzo viene in un primo tempo rifiutato in Ungheria. Ragioni che chiamano in causa "la composizione artistica della materia", la "non riuscita artistica" del romanzo: l'assenza di "bellezza", dunque. Proprio questa presunta assenza di "bellezza" sembra condivisa da Kertész; ed è di estremo

18 I. Kertész, Lo spettatore. Annotazioni 1999-2001, trad. di A. D. Sciacovelli, Bompiani, Milano 2017, p. 154.

${ }^{19}$ I. Kertész, Fiasco (1999), trad. di A. Sciacovelli, Feltrinelli, Milano 2003. 
interesse per noi, dato che può essere letta in parallelo con l'altra affermazione di Kertész: «Non posseggo la parola che salva e redime; non mi ha mai interessato la perfezione, né la bellezza, che non so neanche cosa sia». Si può avanzare l'ipotesi che la bellezza qui contestata sia da intendere come una peculiare categoria storico-estetica (classica) ${ }^{20}$, invalidata dalla violenza delle cose; e che il suo rifiuto avvenga in nome di una diversa riuscita artistica; il che spiegherebbe l'alta considerazione in cui Kertész è tenuto, e il fatto che lo leggiamo.

A proposito di Essere senza destino parla di "composizione atonale"; circa la struttura di Fiasco scrive: «Si tratta, in sostanza, di una struttura musicale»21; questo può spiegare la "diversa riuscita artistica" cui si accennava. Le opere di Kertész (accanto a quelle di Beckett, di Celan e, perché no, di Primo Levi22 ...) restano tra le modalità di sopravvivenza dell'arte (e di una grande arte) "dopo Auschwitz". Dove "arte" vale come simbolo di una "ragione estetica" che si misura con il rischio di naufragio che abbiamo corso, rischio che non è scomparso dai nostri orizzonti. Col "non-bello" dunque.

In una sua notazione leggiamo: «la questione che mi occupava durante la stesura di Essere senza destino era proprio se l'emozionalità del disgusto possa sostituire la simpatia come forza creatrice» ${ }^{23}$. Il negativo attraversato non rende impossibile, intensifica anzi, dà una patina peculiare (anche per Primo Levi e Liliana Segre), alla determinazione di scrivere, di parlarne. Che resta una forma di ripresa di un senso della propria vita. La «prima domanda che ci si deve porre», troviamo scritto altrove, è «se il mondo attribuisce ancora

20 I. Kertész, Dossier K., cit., p. 191. A p. 199 di Diario dalla galera (1992), a cura di A. Melazzini, trad. di K. Sándor, RCS Libri, Milano 2009, leggiamo: «Oggi l'arte classica, ovvero un'arte fondata su una base sociale comune, su una cultura, è impossibile».

${ }^{21}$ A proposito di Fiasco scrive: «Non è un caso che durante la redazione del romanzo io abbia ascoltato così volentieri Il mandarino meraviglioso di Bartók». Lo spettatore, cit., p. 72.

${ }^{22}$ Kertész, quando parla di Levi non mostra tuttavia alcuna simpatia verso di lui: in L'ultimo rifugio, cit., p. 248, ne parla persino come di uno "scrittore sostanzialmente mediocre».

${ }^{23}$ I. Kertész, Diario dalla galera, cit., p. 205. 
un valore alla vita» ${ }^{24}$. La scrittura è una risposta positiva a questo. Positiva, giacché, scrive, «Se la mia fede ingenua nei valori autentici - potrei dire originali - non fosse rimasta intatta, non avrei mai potuto creare nulla» ${ }^{25}$. La "passione" della «scrittura mi è sempre servita a liberarmi dall'oppressione dell'ambiente e delle circostanze» 26 .

La lettura delle pagine di Kertész lascia malgrado tutto un sapore di positività nell'animo, di religiosità non confessionale. «L'Olocausto è valore, perché a costo di immense sofferenze ci ha portato a una conoscenza immensa e di conseguenza esso serba un immenso valore etico». E non riguarda solo i sopravvissuti, che sono «soltanto portatori delle più estreme condizioni dell'uomo dei nostri giorni»; «la sopravvivenza non è solo un problema dei sopravvissuti», riguarda noi tutti, col nostro passato e il nostro futuro dentro 27 .

Un passato, il suo angosciante passato, sopravvive negli scritti di Kertész in una presenza insolita, eppure densa di significatività e di valori. Ed egli dichiara di essere interessato, più che semplicemente a "come vivere" il proprio mondo, a "come rappresentarlo» 28 ; dunque a come testimoniarne scrivendo. Anche per lui l'oblio, la paura e l'angoscia di esso, è il sentimento dominante tra le vittime (e in senso opposto tra i carnefici, che volevano cancellare ogni traccia degli orrori commessi): «Quest'angoscia andava oltre l'orrore, oltre la vita e la morte dei singoli, oltre l'intenso desiderio di giustizia» ${ }^{29}$. Questo riguarda tutti noi, che poniamo la memoria a fondamento dell'idea di Europa che perseguiamo (e della nostra stessa vita) utopicamente forse, ma non utopisticamente.

\footnotetext{
${ }^{24}$ I. Kertész, Il secolo infelice (2007), tr. it. a cura di A. Melazzini e K. Sándor, Bompiani, Milano 2009, p. 121; a p. 129 leggiamo: «una civiltà che non dichiara i propri valori oppure li abbandona imbocca la strada della decadenza».

${ }^{25}$ Ivi, pp. 140-141.

26 Ivi, p. 163.

27 Ivi, pp. 84 e 82.

28 Ivi, p. 93.

${ }^{29}$ Ivi, p. 72; e si vedano le pp. successive, in cui è chiamato a testimone Améry.
} 
La cultura è il supporto imprescindibile della guerra contro l'oblio, ad essa è affidata la salvezza della memoria. E scrivere per lui è un incremento di vita, un modo di reagire all'indifferenza che annichilisce:

ho conquistato abbastanza presto la mia libertà di spirito, e dal momento in cui ho deciso di dedicarmi alla scrittura, i miei problemi ho potuto considerarli, a un tratto, come materia prima della mia arte. E sebbene tale materia prima abbia un aspetto abbastanza tenebroso, la forma la redime e la trasforma in gioia. Infatti è possibile scrivere soltanto attingendo a un'abbondanza di energie, dunque alla gioia; la scrittura - questo non l'ho inventato io - è vita potenziata ${ }^{30}$.

\section{Nota bibliografica}

BAUDELAIRE, Charles, Richard Wagner, tr. it. di L. Merlini, con prefazione di G. Macchia, Passigli, Firenze 1983.

CONATI, Marcello, Rigoletto. Un'analisi drammatico-musicale, Marsilio, Venezia 1992.

—, Verdi. Interviste e Incontri, EDT, Torino 2000.

DAHLHAUS, Carl, I drammi musicali di Richard Wagner, tr. it. a cura di L. Bianconi, Marsilio, Venezia 1984.

DIODATO, Roberto, "La bellezza, qualsiasi cosa sia, non salverà il mondo" [on-line], su Fondazione Corrente Onlus (Facebook). Pubblicato il 10 novembre 2021, consultato il 10 dicembre 2021. Disponibile

${ }^{30}$ I. Kertész, Dossier K., cit., p. 56. A p. 155 di Lo spettatore, cit., "C'è gioia dentro di me, c'è una grave e fruttuosa sofferenza...»; a p. 215, cosciente della precaria sopravvivenza dei suoi libri, e di sé («Tra non molto arriverà per me il tempo di morire»), Kertész annota: «Io però mi sono divertito a scriverli, mi hanno donato l'illusione di aver vissuto una vita piena». A p. 155 di L'ultimo rifugio, cit., coloro che lo vedono come "trascurato", «ignorato", "non si accorgono che faccio il mio lavoro non per costrizione, ma per diletto»; e a p. 244: «La vecchia gioia di scrivere", "scrivere un romanzo è l'unico rifugio per me...». In Liquidazione (2003), tr. it. a cura di A. Sciacovelli, Feltrinelli, Milano 2016, mette in bocca a un protagonista: «Ma io credo nella scrittura. In nient'altro se non nella scrittura. L'uomo vive come un verme, ma scrive come gli dèi», p. 88, e vd. p. 87. 
all'indirizzo: https://www.facebook.com/1408683729436034/videos/319 $\underline{028896324051 /}$.

DOSTOEVSKIJ, Fëdor, L’idiota, introduzione di A. Torno, note di E. Lo Gatto, tr. it. di G. Faccioli e L. Satta Boschian, Bompiani, Milano 2009.

KERTÉSZ, Imre, Essere senza destino (1975), tr. it. di B. Griffini, Feltrinelli, Milano 2004.

—, Diario dalla galera (1992), a cura di A. Melazzini, trad. di K. Sándor, RCS Libri, Milano 2009.

—, Fiasco (1999), trad. di A. Sciacovelli, Feltrinelli, Milano 2003.

—, Liquidazione (2003), tr. it. a cura di A. Sciacovelli, Feltrinelli, Milano 2016.

—, Dossier K. (2006), trad. di M. D’Alessandro, Feltrinelli, Milano 2009.

—, Il secolo infelice (2007), tr. it. a cura di A. Melazzini e K. Sándor, Bompiani, Milano 2009.

—, L’ultimo rifugio. Romanzo di un diario (2014), trad. di M. Sciglitano, Bompiani, Milano 2016.

—, Lo spettatore. Annotazioni 1999-2001, trad. di A. D. Sciacovelli, Bompiani, Milano 2017.

LISZT, Franz, Lohengrin e Tannhäuser di Richard Wagner (1854), tr. it. di R. Morello, introduzione di P. Isotta, Mondadori, Milano 1983.

PRINCIPE, Quirino, "Il bianco e l'argento di Lohengrin", in Lohengrin, Programma di Sala, Edizioni del Teatro alla Scala, Milano 2006.

—, Lohengrin, Jaca Musica, Milano 2012. 
—, Wagner e noi, Jaca Musica, Milano 2012.

SCARAMUZZA, Gabriele, Scelte, Mimesis, Milano 2021.

WAGNER, Cosima, La mia vita a Bayreuth 1883/1930, a cura di D. Mack, tr. it. di U. Gandini, Rusconi, Milano 1982.

WAGNER, Richard, La mia vita, tr. it. di M. Mila, EDT, Torino 1983.

—, Mein Leben, Schünemann, Bremen 1986.

\section{Nota biografica}

Gabriele Scaramuzza si è laureato in Filosofia a Pavia. Ha insegnato estetica a Padova, Verona, Sassari, Milano. Tra le sue principali pubblicazioni: Le origini dell'estetica fenomenologica; Antonio Banfi, la ragione e l'estetico (Antenore, Padova 1976), Il brutto all'opera. L'emancipazione del negativo nel teatro di Giuseppe Verdi (Mimesis, Milano 2013), Kafka a Milano. La città, la testimonianza, la legge (Mimesis, MilanoUdine 2013), Incontri. Per una filosofia della cultura (Mimesis, Milano-Udine 2017). A ciò vanno aggiunti gli scritti autobiografici In fondo al giardino. Ritagli di memorie (2015) e Un’insostenibile voglia di vivere. Frammenti di memorie e riflessioni (2017), entrambi editi da Mimesis. 